\title{
Effect of Antiseptic Agents on the Surface Microhardness of Calcium Silicate-Based Materials
}

\author{
Sudo Susumu ${ }^{1}$, Handa Keisuke ${ }^{1,2 *}$, Kanehira Masafumi ${ }^{1}$, Yahata Yoshio ${ }^{1}$ and Saito Masahiro ${ }^{1}$ \\ ${ }^{1}$ Division of Operative Dentistry, Department of Restorative Dentistry, Tohoku University Graduate School of Dentistry, Japan \\ ${ }^{2}$ Division of Molecular Biology and Oral Biochemistry, Department of Oral Science, Graduate School of Dentistry, Kanagawa \\ Dental University, Japan
}

*Corresponding author: Keisuke Handa, Division of Molecular Biology and Oral Biochemistry, Department of Oral Science, Graduate School of Dentistry, Kanagawa Dental University, 82 Inaoka-cho, Yokosuka, Kanagawa 238-8580, Japan.

\section{ARTICLE INFO}

Received: May 20, 2020

Published: 幽 June 09, 2020

Citation: Sudo S, Handa K, Kanehira M, Yahata Y, Saito M. Effect of Antiseptic Agents on the Surface Microhardness of Calcium Silicate-Based Materials. Biomed J Sci \& Tech Res 28(2)-2020. BJSTR. MS.ID.004610.

Abbreviation: CSMs: Calcium Silicate-Based Materials; MTA: Mineral Trioxide Aggregate; PMTA: Pro Root MTA; ERRM: Endo Sequence BC Root Repair Material Putty; EMTA: Endoseal MTA; CHX: Chlorhexidine Gluconate; SEM: Scanning Electron Microscopy; XRD: X-ray Diffraction
ABSTRACT

Purpose: We evaluated the effects of antiseptic agents on the surface microhardness of calcium silicate-based materials (CSMs).

Methods: Cylindrical molds were filled with Pro Root MTA (Dentsply Tulsa Dental, Tulsa, OK, USA), Endo Sequence BC Root Repair Material Putty (ERRM; Brasseler USA, Savannah, GA, USA), or Endoseal MTA (Maruchi, Wonju, Korea). The CSMs were covered with a wet cotton pellet, soaked with distilled water (DW), $2 \%$ chlorhexidine gluconate (CHX), and 70\% ethanol. The surface microhardness was measured after setting.

Result: After 1 week, no microhardness was evident in any CSM with CHX, or in ERRM with ethanol, but was measurable in all CSMs with DW. The surface microhardness of CSMs is affected by contact with CHX and ethanol.

Conclusion: Insufficiently set layers formed on a CSM surface can result in a decreased microhardness. Further studies are required to establish procedures for the appropriate removal of insufficient setting layers in a clinical context.

Keywords: Calcium Silicate-Based Materials; Chlorhexidine Gluconate; Ethanol; Surface Microhardness; Scanning Electron Microscope

\section{Introduction}

Mineral trioxide aggregate (MTA), which was originally derived from Portland cement, has been developed as a material for perforation repair and root-end filling [1,2]. Calcium silicatebased materials (CSMs), including MTA, are chemically stable materials with a high physical strength and alkaline $\mathrm{pH}$ that confer antimicrobial properties [3]. CSMs have now been used for vital pulp therapy, regenerative endodontics, root filling, root resorption, and other applications due to their high biocompatibility and sealing ability $[4,5]$. The physical properties of CSMs are influenced by their contact with blood during their setting. A previous bacterial leakage study of root-end cavities filled using Pro Root MTA (PMTA; Dentsply Tulsa Dental, Tulsa, OK, USA) indicated that more leakage occurred from PMTA fillings contaminated with saliva than with saline or blood [6]. Another report of the push-out bond strength of simulated furcation perforation repair using PMTA or CEM
(Bionique Dent, Tehran, Iran) showed decreased strength in cases of blood contamination compared to those without contamination [7]. Contact with blood during setting for PMTA, Ortho MTA (Bio MTA, Seoul, Korea) and Retro MTA (BioMTA, Seoul, Korea) has been further shown to adversely affect their surface microhardness [8].

In addition, prior studies have demonstrated that the acidic environment provided by exposing pus from a periapical abscess can negatively affect the physical properties of CSMs in terms of microleakage prevention [9] and the push-out bond strength $[10,11]$. These previous investigations have indicated that the prognosis of root canal treatment can be impacted by a decline in the physical properties of CSMs through environmental exposure to saliva, blood or pus. Many types of antiseptic have been used for root canal irrigation and from inside the pulp chamber these agents can make contact with CSMs and affect their physical properties. 
Chlorhexidine gluconate (CHX) is an alternative root canal irrigant to $\mathrm{NaOCl}$ that is used because of its lower cytotoxicity towards periapical tissue [12] and antimicrobial potency for up to 12 weeks [13]. However, CHX may also decrease the push-out bond strength of CSMs even when used for only 30 minutes after filling [14]. Ethanol can be used on the other hand to remove root canal sealer remnants [15]. The setting properties of CSMs remain unclear over long-term contact with irrigants that sustain their pharmacological action inside the root canal over days or weeks. Since the physical strength of CSMs is decreased when setting under dry conditions [16], a moist environment has been recommended when using these compounds through the placement of a wet cotton pellet soaked with distilled water (DW) [17]. Antiseptic agents can also be used to moisten the cotton pellet if an antibacterial regimen is required. We therefore sought to clarify the long-term effects of contact with antiseptic agents under setting conditions on the physical properties of CSMs. We thus investigated the effects of $2 \%$ CHX or $70 \%$ ethanol on the CSM surface microhardness. In addition, the CSM surfaces were observed by scanning electron microscopy (SEM), and their crystalline structures was analyzed by X-ray diffraction (XRD), to assess the morphological impacts of this contact with CHX and ethanol.

\section{Materials and Methods}

\section{Specimen Preparation}

Cylindrical molds with a diameter of $5 \mathrm{~mm}$ and a depth of 5 $\mathrm{mm}$ were formed on an acrylic bar. The molds were then filled with three different CSMs (PMTA, EndoSequence BC Root Repair Material Putty [ERRM; Brasseler USA, Savannah, GA, USA], or Endoseal MTA [EMTA; Maruchi, Wonju, Korea]). PMTA was mixed with DW in accordance with the manufacturer's instructions. The premixed ERRM and EMTA preparations were then used to directly fill the molds. After filling by these materials, the bottom surface was immersed in PBS. A wet cotton pellet soaked with DW, 2\% CHX or $70 \%$ ethanol was placed on the upper surface of the CSMs and fixed in place with hydraulic temporary sealing material (Caviton; GC, Tokyo, Japan). The specimens were then placed in an incubator at $37^{\circ} \mathrm{C}$ with $100 \%$ humidity (Figure 1 ).

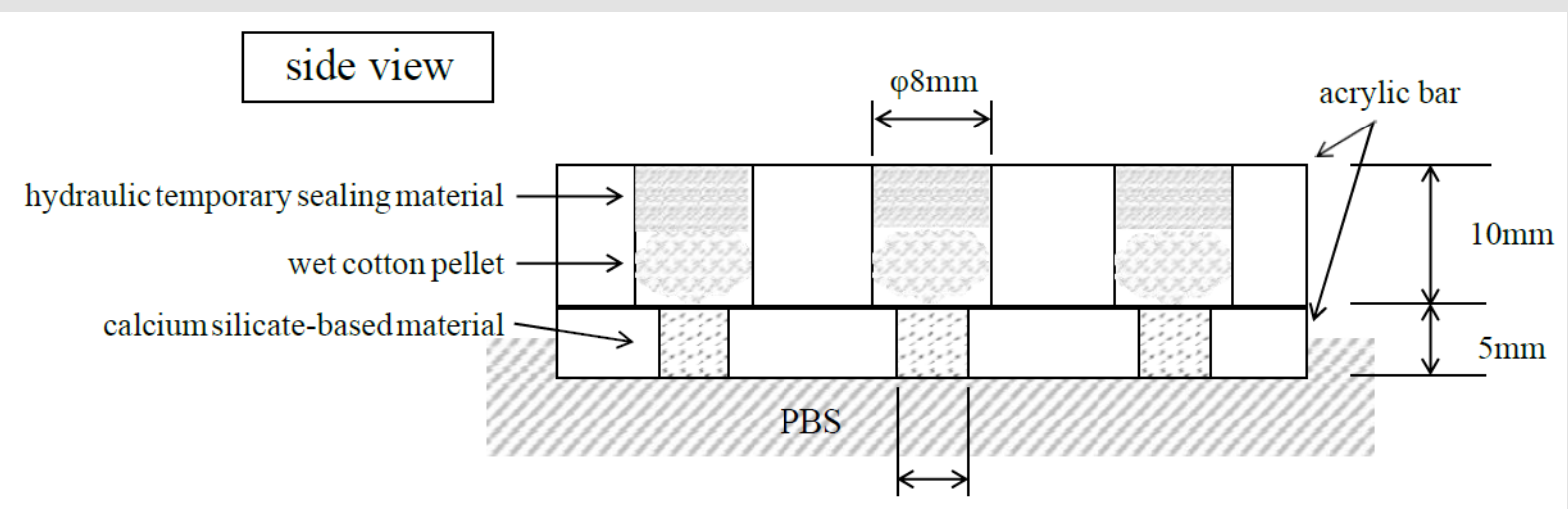

Figure 1: Schema for the experimental apparatus used in this study. Calcium silicate-based materials were covered with wet cotton pellets and hydraulic temporary sealing material. The pellets were then soaked with either distilled water, $2 \%$ chlorhexidine gluconate or $70 \%$ ethanol. The bottom of the calcium silicate-based materials was immersed in PBS.

\section{Surface Microhardness Test}

At 1 and 4 weeks after setting, the surface microhardness of the CSM specimens was determined using a micro hardness tester (HM102; Mitutoyo, Kanagawa, Japan). Before this testing, the surfaces of the specimens were wet ground with silicon carbide paper $(600$, 800,1000 grit) at room temperature. Indentations were made in each specimen with a Knoop diamond indenter set at 10 gf and a $15 \mathrm{sec}$ dwell time. Five measurements were made on the surface of each specimen in the molds, with the average value taken as the hardness. According to our pilot study, the surface microhardness does not differ with or without the molds. There were five specimens for each CSM in this testing (PMTA, ERRM, EMTA), three solutions (DW, CHX, 70\% ethanol) and two setting periods (1 or 4 weeks), totaling a sample size of 90 . Statistical analysis included 2-way analysis of variance and a Tukey multiple comparison post hoc test, with a significance level set at $\alpha=.05$.

\section{Scanning Electron Microscopy}

The same CSM specimens used in the surface microhardness test were immersed in each solution. After 1 or 4 weeks of setting, the surfaces of these CSMs were coated with platinum and analyzed with a scanning electron microscope (VE-8800, KEYENCE, Osaka, Japan).

\section{X-Ray Diffraction Analysis}

The crystalline structures of the CSMs were analyzed by X-ray diffraction (XRD) after immersion in each test solution for 1 week. The XRD patterns were recorded using an X-ray diffractometer ( $R$ Smart Lab, Rigaku, Tokyo, Japan) with CuK $\beta$ radiation, which was generated at $45 \mathrm{kV}$ and $200 \mathrm{~mA}$. The X-ray beam angle $2 \theta(\mathrm{deg})$ range was set at between 15 to $60 \mathrm{deg}$ and scanned at $10 \mathrm{deg} /$ min. The XRD patterns for the specimens were compared and matched with those in the ICDD database (International Center for Diffraction Data, Newtown Square, PA, USA). 


\section{Result}

\section{CSM Surface Microhardness}

The mean CSM surface microhardness values between the different experimental groups are presented in Table 1. After 1 week of setting, the surface microhardness could not be measured in any of the CSMs with CHX or in ERRM with ethanol. This was because the indentations of these samples were not readable due to an insufficient surface microhardness to withstand the force produced by the indenter. After 4 weeks of setting however, the surface microhardness of PMTA and ERRM could be determined in the CHX group, although that of EMTA could not. In contrast, the surface microhardness could be measured in all of the CSMs with DW after 1 week of setting. In the statistical analysis of these results, EMTA showed a significantly lower surface microhardness than either PMTA or ERRM $(\mathrm{P}<.05)$. Notably however, there was no significant difference observed between the two setting periods. PMTA and ERRM surface microhardness values could be measured following contact with all solutions after 4 weeks of setting. The statistical analysis of these findings indicated that PMTA had a significantly greater surface microhardness than ERRM $(\mathrm{P}<.05)$, but there was no significant difference between the materials that contacted DW, CHX and 70\% ethanol (Table1).

Table 1: Surface microhardness (Knoop hardness) measurements for the CSM specimens under the indicated conditions Values shown are the mean \pm standard deviation. BML, below measurement limit. Different letters (uppercase between each of the distilled water specimens, lowercase between the Pro Root MTA and Endo Sequence BC RRM specimens at 4 weeks) indicate a significant difference $(\mathrm{P}<.05)$.

\begin{tabular}{|c|c|c|c|c|}
\hline & Setting Time & Distilled Water & 2\% Chlorhexidine & $70 \%$ Ethanol \\
\hline \multirow{2}{*}{ Pro Root MTA } & 1 week & $33.5 \pm 1.2 \mathrm{~A}$ & BML & $37.4 \pm 2.2$ \\
\hline & 4 weeks & $48.2 \pm 4.3 \mathrm{Aa}$ & $53.5 \pm 3.5$ a & $54.7 \pm 16.7$ a \\
\hline \multirow{2}{*}{ Endo Sequence BC RRM } & 1 week & $47.8 \pm 7.8 \mathrm{~A}$ & BML & BML \\
\hline & 4 weeks & $39.2 \pm 2.8 \mathrm{Ab}$ & $32.3 \pm 3.5 \mathrm{~b}$ & $40.2 \pm 6.2 \mathrm{~b}$ \\
\hline \multirow{2}{*}{ Endoseal MTA } & 1 week & $5.5 \pm 0.3 \mathrm{~B}$ & BML & $4.6 \pm 0.1$ \\
\hline & 4 weeks & $7.4 \pm 0.5 \mathrm{~B}$ & BML & $6.5 \pm 1.1$ \\
\hline
\end{tabular}

\section{Scanning Electron Microscopy of the CSM Surfaces}

Cubic-like crystals were observed over each CSM surface that underwent DW immersion. Stratified structures were observed on the orthogonal plane of the smooth-surface plane (Figure 2A-F). Particulate precipitates were observed on the EMTA crystal surface (Figure 2C and F) and scarious crystals were detectable over each CSM surface that had undergone CHX immersion (Figure 2G-L). The crystal morphologies under DW and CHX immersion conditions showed no differences after 1 or 4 weeks of setting, although the crystal size had increased slightly at the 4-week time point. Cotton wool-like structures were observed over each CSM surface at 1 week of setting in the $70 \%$ ethanol immersion group. The density and sizes of the ERRM crystals were lower than those of PMTA and EMTA (Figure 2M-0). At 4 weeks of setting in the 70\% ethanolimmersed materials, PMTA and EMTA showed clusters of small cubic crystals (Figure 2P and R). The density and size of the cotton wool-like structures seen for ERRM were increased at 4 weeks compared with 1 week of setting (Figure 2Q).

\section{X-Ray Diffraction Analysis}

XRD was performed to determine the effects on setting in CSMs after their exposure to moisture from DW, CHX or $70 \%$ ethanol. The XRD patterns of the CSMs at 1 week were different in each type of solution. In the DW-immersed PMTA, the peaks were consistent with calcite $\left(\mathrm{CaCO}_{3}\right.$, ICDD \# 01-072-1937), bismuth oxide $\left(\mathrm{Bi}_{2} \mathrm{O}_{3}\right.$, ICDD \# 01-071-0465), portlandite (Ca[OH] $]_{2}$ ICDD \# 01-070- 5492), and larnite ( $\mathrm{Ca}\left[\mathrm{SiO}_{4}\right]$, ICDD \# 01-076-3608) (Figure 3A). In the CHXimmersed PMTA, the peaks were consistent with calcium hydroxide ( $\mathrm{Ca}[\mathrm{OH}]_{2}$, ICDD \# 01-084-1265), bismuth oxide ( $\mathrm{Bi}_{2} \mathrm{O}_{3}$, ICDD \# 01071-0465), calcium carbonate ( $\mathrm{CaCO}_{3}$, ICDD \# 01-080-9776), and calcium silicate $\left(\mathrm{Ca}_{3} \mathrm{SiO}_{5}\right.$, ICDD \# 00-055-0738) (Fig. 3D). In the ethanol-immersed PMTA, the peaks were consistent with bismuth oxide ( $\mathrm{Bi}_{2} \mathrm{O}_{3}$, ICDD \# 01-071-0465), calcite ( $\mathrm{CaCO}_{3}$, ICDD \# 01-0721937), and calcium silicate $\left(\mathrm{Ca}_{3} \mathrm{SiO}_{5}, \mathrm{ICDD} \#\right.$ 00-055-0738) (Figure $3 G)$. In the DW-immersed ERRM, the peaks were consistent with calcite $\left(\mathrm{CaCO}_{3}\right.$, ICDD \# 00-047-1743) and aluminum phosphide (AlP, ICDD \# 00-012- 0470) (Figure 3B). There was however no consistency among the ethanol-immersed materials in the CHXimmersed ERRM (Figure 3E).

In the ethanol-immersed ERRM, the peaks were consistent with calcite $\left(\mathrm{CaCO}_{3}\right.$, ICDD \# 01-086-2334) and baddeleyite $\left(\mathrm{ZrO}_{2}\right.$, ICDD \# 00-037-1484) (Figure 3H). In the DW-immersed EMTA, the peaks were consistent with baddeleyite (ZrO ${ }^{2}$, ICDD \# 00-037-1484) and calcium carbonate ( $\mathrm{CaCO}_{3}$, ICDD \# 01-080-9775) (Figure 3C). In the CHX-immersed EMTA, the peaks were consistent with baddeleyite ( $\mathrm{ZrO}^{2}$, ICDD \# 00-037-1484), bismuth oxide $\left(\mathrm{Bi}_{2} \mathrm{O}_{3}\right.$, ICDD \# 01-0708245), and bismuth silicate (Bi2SiO5, ICDD \# 00-036-0288) (Figure $3 F$ ). In the ethanol-immersed EMTA, the peaks were consistent with baddeleyite $\left(\mathrm{ZrO}_{2}\right.$, ICDD \# 00-070-2491), calcite ( $\mathrm{CaCO}_{3}$, ICDD \# 01- 078-3262), and ettringite $\left(\mathrm{Ca}_{6} \mathrm{Al}_{2}\left[\mathrm{SO}_{4}\right]_{3}[\mathrm{OH}]_{12}\left[\mathrm{H}_{2} \mathrm{O}\right]_{26}\right)$, ICDD \# 01-075-7554) (Figure 3I). 


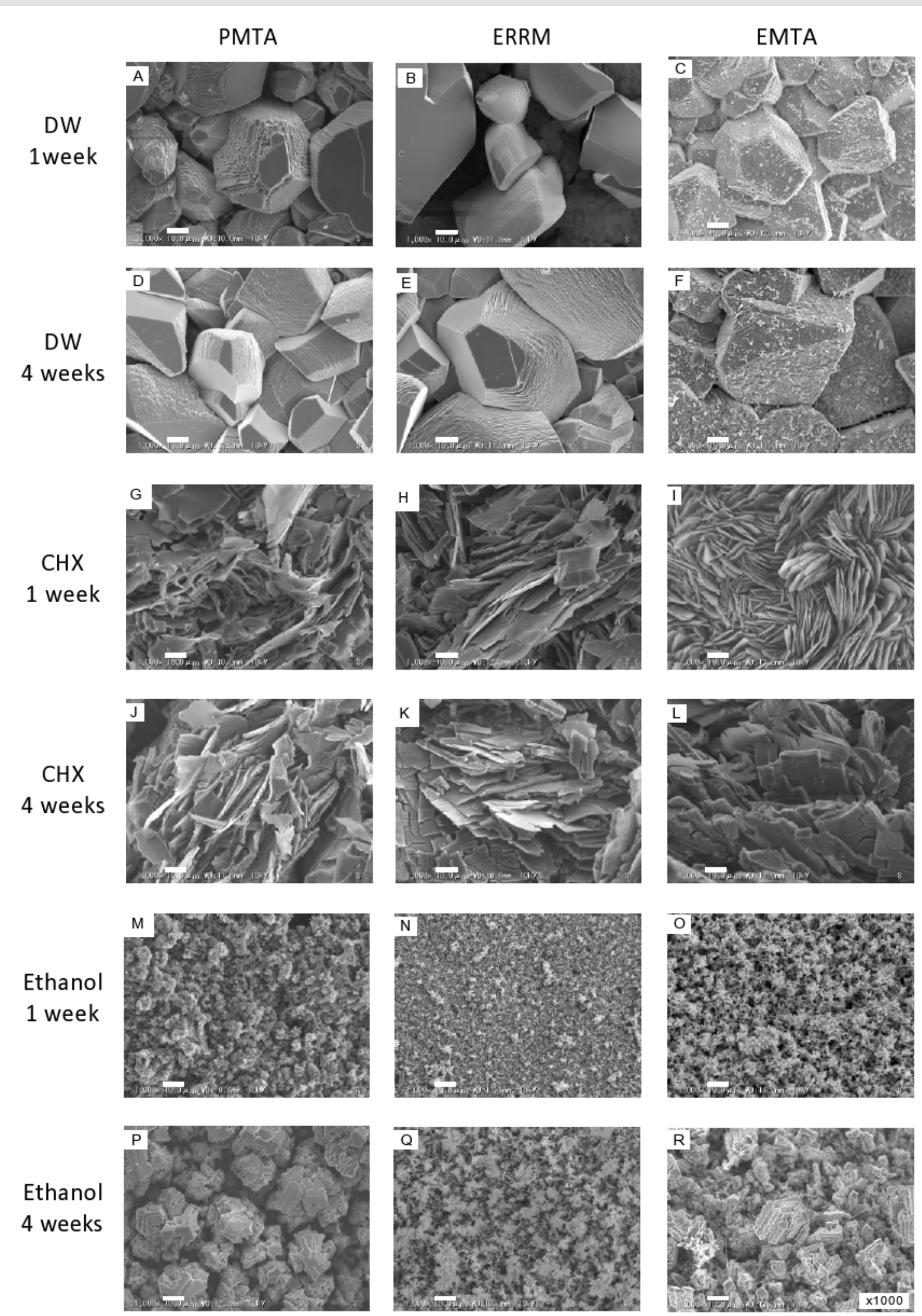

Figure 2: Scanning electron microscope images of CSM surfaces. ProRoot MTA (PMTA: A, D, G, J, M and P), Endo Sequence BC RRM (ERRM: B, E, H, K, N and Q) and Endoseal MTA (EMTA: C, F, I, L, O and R) were immersed in either distilled water (A-F), 2\% chlorhexidine gluconate (G-L) or 70\% ethanol (M-R). Results after 1 week (1W: A-C, G-I and M-O) and 4 weeks (4W: D-F, J-L and P-R) of setting in each solution group are shown. Scale bars, $10 \mu \mathrm{m}$. 

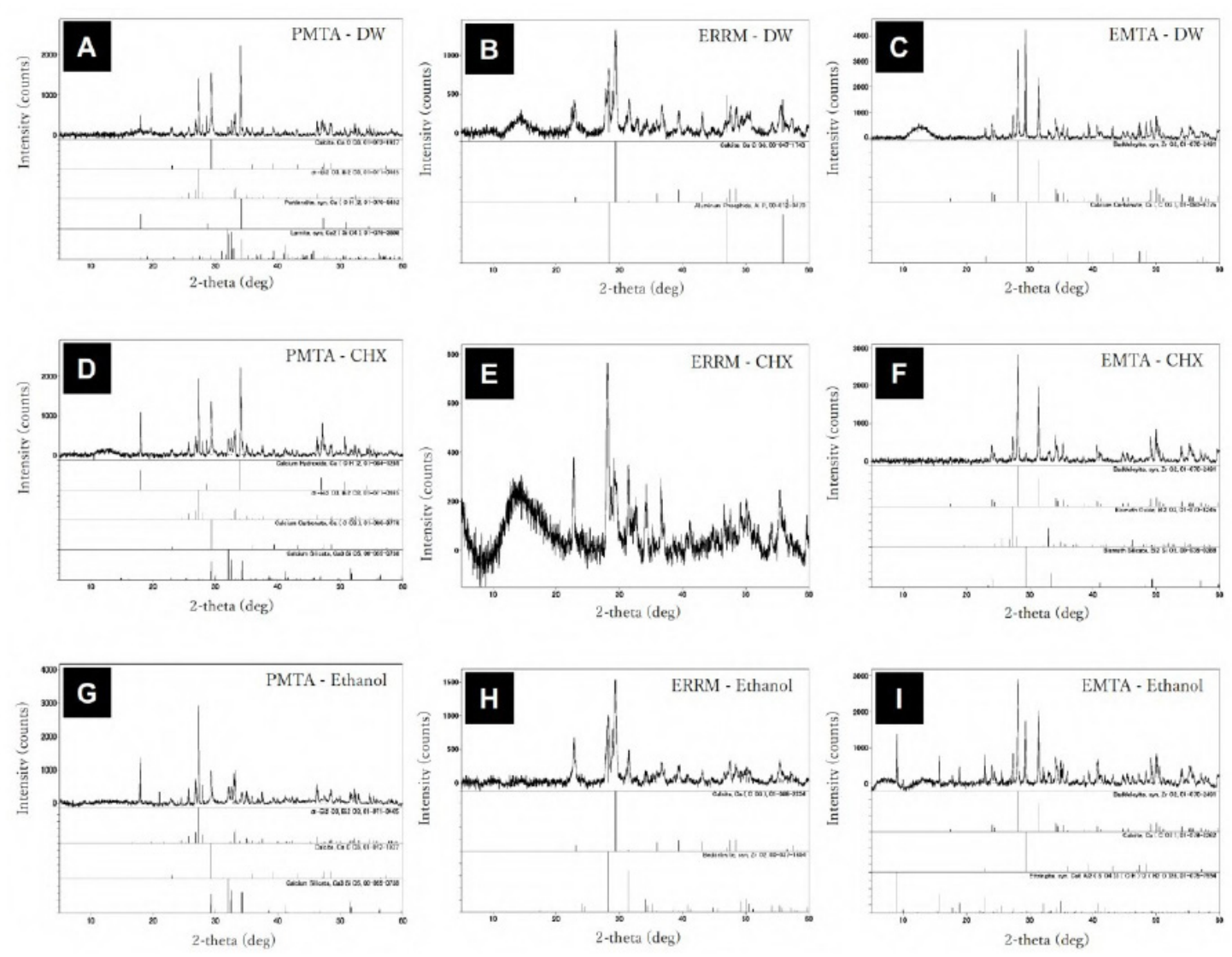

Figure 3: XRD patterns of C SMs after 1 week of setting. Pro Root MTA (PMTA) immersed in distilled water (DW: A), 2\% chlorhexidine gluconate (CHX: D) or 70\% ethanol (Ethanol: G). Endo Sequence BC RRM (ERRM) immersed in distilled water (DW: B), 2\% chlorhexidine gluconate (CHX: E) or 70\% ethanol (Ethanol: H). Endoseal MTA (EMTA) immersed in distilled water (DW: C), $2 \%$ chlorhexidine gluconate (CHX: F) or 70\% ethanol (Ethanol: I).

\section{Discussion}

We have found in our present study that the surface microhardness of CSMs is affected by long-term contact with either $2 \%$ CHX or $70 \%$ ethanol. The degree of setting after 1 week was insufficient in PMTA and ERRM that had contacted with CHX, or in ERRM that had contacted with $70 \%$ ethanol. At the 4 -week time point however, all specimens were substantially set except for EMTA. These results are in accordance with previous findings that the hydration of CSMs progresses continuously in contact with water, with an increasing surface microhardness $[3,18]$. Our current data thus suggest that the insufficient setting of a CSM surface after 1 week when there is contact with either $2 \%$ CHX or ethanol can be recovered over a longer-term setting period. EMTA exposed to CHX still had insufficient setting after 4-weeks, however. PMTA and ERRM were developed as perforation repair and root-end filling materials, whereas EMTA was produced as a root canal sealer. The Vickers microhardness of non-sealer CSMs was previously reported to be 40 or more, whilst that of sealer CSMs was measured at 20 or less [18] indicating that the CSMs of sealers are more affected by exposed solutions. An expansion of setting CSMs was observed previously above the mold [19].
We observed this also in our present study, with some of the protruding surface showing insufficient setting. Before performing a surface microhardness test for CSMs, it is necessary to polish their surface so that it is parallel to the sample table of the microhardness tester, and indentations by a Knoop diamond indenter are clear. Any insufficient setting layers were removed by this polishing treatment so that the exposed hardened layers could be measured. Our present data strongly suggest that insufficient setting layer of the CSM surface affects the surface microhardness. Further studies are required to establish an appropriate way to remove this insufficient set material.

Initially, our observations of the CSM surfaces were performed after polishing but the polished surface was covered with smear and the original crystalline structures were lost. Hence, we analyzed non-polished surfaces in order to observe the original crystalline structure. A cubic crystalline formation was observed in the CSMs that made contact with DW, which is consistent with a previous study [20]. Scarious structures and cotton wool-like structures were observed following contact with CHX and ethanol, respectively. A prior study of SEM observations of a PMTA surface exposed to DW, $\mathrm{NaOCl}$ or $\mathrm{CHX}$ for 30 minutes also described cubic 
crystalline structures in all solutions, but reported that the sizes of the crystals in the CHX group were about $90 \%$ smaller than those in the DW or $\mathrm{NaOCl}$ groups [14]. The scarious structures we here observed in the materials exposed to CHX may have contributed to crystallization due to the long period of contact in our experiments. In the CSMs exposed to $70 \%$ ethanol, the cubic crystalline structures were smaller than those in the DW group, and cotton wool-like structures were observed. The crystalline formation of ERRM with ethanol was delayed compared to PMTA or EMTA, which may underlie why the surface microhardness of ERRM was not measurable following contact with ethanol.

Although there has to our knowledge been no prior report on the influence of ethanol on CSMs, the dehydration effects of ethanol may have prevented sufficient water from reaching the ERRM material. Our current data suggest that long-term contact with a solution affects the surface microhardness of a CSM, and that any reagent that inhibits hydration will adversely impact the setting of these materials. Although there was no significant difference in the surface microhardness of PMTA and ERRM after 4 weeks of setting, the morphology of the crystals was different in each solution. Because the surface microhardness was measured after polishing, differences in the crystal morphology before polishing might not be reflected in the results. We could not therefore completely correlate the SEM image before polishing with the surface microhardness after polishing.

The precipitation of calcium hydroxide on CSMs was not identified in the XRD spectrum analysis except for the DW- and CHX-immersed PMTA. Previous studies have produced inconsistent results regarding the identification of calcium hydroxide, a known product of hydration reactions in CSMs [14, 21]. Furthermore, radiopaque agents in the ERRM were not identified in all solution groups. XRD spectrum analysis is a method of identifying crystalline structures through their peak patterns but provides only onedimensional information, and reflections with similar or the same interplanar distance (d values) are overlapped. In complex materials such as CSMs, the extent of this overlap is very severe. For complex structural problems, such as peak overlaps, impurities, disorder, and aperiodic structures, it is not possible to achieve a complete assessment of the materials using a single technique [22].

Previous studies have also reported a relationship between the physical properties and the crystalline structure of CSMs, but the nature of this association is still unclear $[14,20,23]$. Similar to previous studies, we could not clarify the relationship between the surface microhardness and crystalline structure of CSMs in our present analyses. The antibacterial action of CSMs are due to their high $\mathrm{pH}$ [3], but although mixing with 2\% CHX could be expected to enhance the antibacterial action of PMTA, this mixture did not set even after 1 week [24]. However, there was no significant difference found previously between the compressive strengths of CSMs following mixing with DW and either $0.2 \%$ or $0.12 \%$ CHX
[25]. These findings have suggested that the concentration of CHX should be less than $0.2 \%$ when used in conjunction with CSM filling. Furthermore, when used for root canal irrigation, the antibacterial action of 2\% CHX has been found to persist for 90 days [26]. It must be noted however that the intra-canal residual concentration of CHX after immersion at $2 \%$ for 10 minutes is reported to decrease to $0.0048 \%$ [27], indicating that CSM filling should be performed 10 minutes after a $2 \%$ CHX irrigation.

\section{Conclusion}

The surface microhardness of CSMs is affected by contact with either $2 \%$ CHX or $70 \%$ ethanol after 1 week of setting. Distilled water should therefore be used for wetting the cotton pellet that covers these filling materials. An insufficient setting layer on a CSM surface will cause a decline in its microhardness. Further studies are required to establish an appropriate clinical protocol for removal of insufficiently set CSMs.

\section{Acknowledgments}

This work was supported by Japan Society for the Promotion of Science (JSPS) KAKENHI, Grant Number 18H02975 and 18K09592.

\section{Conflict of interest}

The authors declare no conflict of interest associated with this study.

\section{References}

1. Lee SJ, Monsef M, Torabinejad M (1993) Sealing ability of a mineral trioxide aggregate for repair of lateral root perforations. J Endod 19: 541-544.

2. Torabinejad M, Watson TF, Pitt Ford TR (1993) Sealing ability of a mineral trioxide aggregate when used as a root end filling material. J Endod 19: 591-595.

3. Parirokh M, Torabinejad M (2010) Mineral trioxide aggregate: a comprehensive literature review-Part I: chemical, physical, and antibacterial properties. J Endod 36:16-27.

4. Parirokh M, Torabinejad M, Dummer PMH (2018) Mineral trioxide aggregate and other bioactive endodontic cements: an updated overview - part I: vital pulp therapy. Int Endod J 51:177-205.

5. Torabinejad M, Parirokh M, Dummer PMH (2018) Mineral trioxide aggregate and other bioactive endodontic cements: an updated overview - part II: other clinical applications and complications. Int Endod J 51: 284-317.

6. Montellano AM, Schwartz SA, Beeson T (2006) Contamination of toothcolored mineral trioxide aggregate used as a root-end filling material: a bacterial leakage study. J Endod 32: 452-455.

7. Rahimi S, Ghasemi N, Shahi S, Lotfi M, Froughreyhani M, et al. (2013) Effect of blood contamination on the retention characteristics of two endodontic biomaterials in simulated furcation perforations. J Endod 39: 697-700.

8. Song M, Yue W, Kim S, Kim W, Kim Y, et al. (2016) The effect of human blood on the setting and surface micro-hardness of calcium silicate cements. Clin Oral Investig 20: 1997-2005.

9. Saghiri MA, Lotfi M, Saghiri AM, Vosoughhosseini S, Fatemi A, etal. (2008) Effect of $\mathrm{pH}$ on sealing ability of white mineral trioxide aggregate as a root-end filling material. J Endod 34: 1226-1229. 
10. Shokouhinejad N, Nekoofar MH, Iravani A, Kharrazifard MJ, Dummer PM (2010) Effect of acidic environment on the push-out bond strength of mineral trioxide aggregate. J Endod 36:871-874.

11. Bolhari B, Nekoofar MH, Sharifian M, Ghabrai S, Meraji N, Dummer PM (2014) Acid and microhardness of mineral trioxide aggregate and mineral trioxide aggregate-like materials. J Endod 40: 432-435.

12. Gonçalves LS, Rodrigues RC, Andrade Junior CV, Soares RG, Vettore MV (2016) The effect of sodium hypochlorite and chlorhexidine as irrigant solutions for root canal disinfection: A systematic review of clinical trials. J Endod 42: 527-532.

13. Mohammadi Z, Abbott PV (2009) Antimicrobial substantivity of root canal irrigants and medicaments: a review. Aust Endod J 35: 131-139.

14. Hong ST, Bae KS, Baek SH, Kum KY, Shon WJ, et al. (2010) Effects of root canal irrigants on the push-out strength and hydration behavior of accelerated mineral trioxide aggregate in its early setting phase. J Endod 36: 1995-1999.

15. Kuga MC, Faria G, Rossi MA, Do Carmo Monteiro JC, Bonetti-Filho I, et al. (2013) Persistence of epoxy-based sealer residues in dentin treated with different chemical removal protocols. Scanning 35: 17-21.

16. Shokouhinejad N, Jafargholizadeh L, Khoshkhounejad M, Nekoofar MH Raoof M (2014) Surface microhardness of three thicknesses of mineral trioxide aggregate in different setting conditions. Restor Dent Endod 39: 253-257.

17. Torabinejad M, Chivian N (1999) Clinical application of mineral trioxide aggregate. J Endod 25: 197-205.

18. Guo YJ, Du TF, Li HB, Shen Y,Mobuchon C, et al. (2016) Physical properties and hydration behavior of a fast setting bioceramic endodontic material. BMC Oral Health 20: 16:23.

ISSN: 2574-1241

DOI: 10.26717/BJSTR.2020.28.004610

Handa Keisuke. Biomed J Sci \& Tech Res

(C) This work is licensed under Creative Commons Attribution 4.0 License

Submission Link: https://biomedres.us/submit-manuscript.php
19. Walsh RM, Woodmansey KF, Glickman GN, He J (2014) Evaluation of compressive strength of hydraulic silicate-based root-end filling materials. J Endod 40: 969-972.

20. Lee YL, Lee BS, Lin FH, Yun Lin A, Lan WH, et al. (2004) Effects of physiological environments on the hydration behavior of mineral trioxide aggregate. Biomaterials 25: 787-793.

21. Han L, Okiji T, Okawa S (2010) Morphological and chemical analysis of different precipitates on mineral trioxide aggregate immersed in different fluids. Dent Mater J 29: 512-517.

22. Li J, Sun J (2017) Application of X-ray diffraction and electron crystallography for solving complex structure problems. Acc Chem Res 50: 2737-2745.

23. Guneser MB, Akbulut MB, Eldeniz AU (2013) Effect of various endodontic irrigants on the push- out bond strength of biodentine and conventional root perforation repair materials. J Endod 39:380-384.

24. Kogan P, He J, Glickman GN, Watanabe I (2006) The effects of various additives on setting properties of MTA. J Endod 32: 569-572.

25. Bidar M, Eslami N, Naghavi N, Fasihi Z, Attaran Mashhadi N (2015) The effect of different concentrations of chlorhexidine gluconate on the compressive strength of mineral trioxide aggregate. J Dent Res Dent Clin Dent Prospects 9: 1-5.

26. Baca P,Junco P,Arias-Moliz MT, Castillo F, Rodríguez-Archilla et al. (2012) Antimicrobial substantivity over time of chlorhexidine and cetrimide. J Endod 38: 927-930.

27. Rosenthal S, Spångberg L, Safavi K (2004) Chlorhexidine substantivity in root canal dentin. Oral Surg Oral Med Oral Pathol Oral Radiol Endod 98: 488-492.

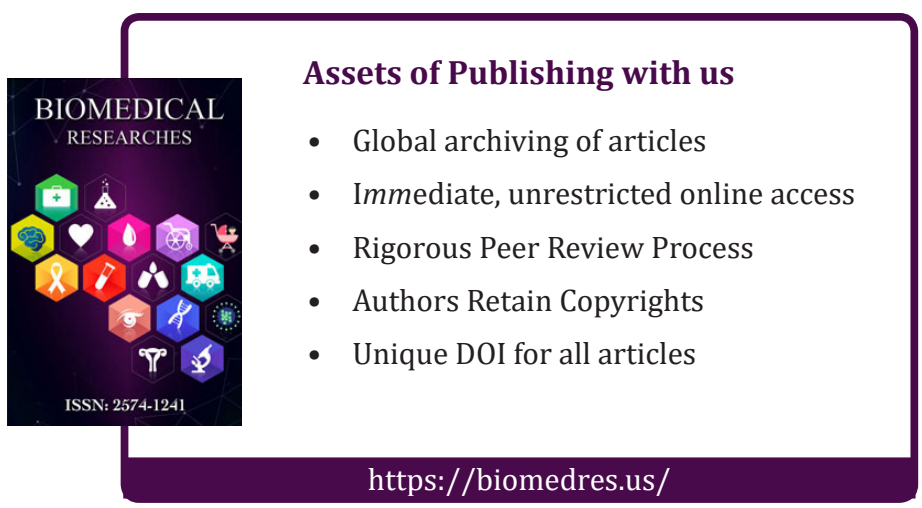

